\title{
Temporal RDF
}

\author{
Claudio Gutierrez ${ }^{1}$, Carlos Hurtado ${ }^{1}$, and Alejandro Vaisman ${ }^{2}$ \\ 1 Department of Computer Science, \\ Universidad de Chile \\ \{cgutierr, churtado\}@dcc.uchile.cl \\ 2 Department of Computer Science, \\ Universidad de Buenos Aires, \\ avaisman@dc.uba.ar
}

\begin{abstract}
The Resource Description Framework (RDF) is a metadata model and language recommended by the W3C. This paper presents a framework to incorporate temporal reasoning into RDF, yielding temporal RDF graphs. We present a semantics for temporal RDF graphs, a syntax to incorporate temporality into standard RDF graphs, an inference system for temporal RDF graphs, complexity bounds showing that entailment in temporal RDF graphs does not yield extra asymptotic complexity with respect to standard RDF graphs and sketch a temporal query language for RDF.
\end{abstract}

\section{Introduction}

The Resource Description Framework (RDF) [14 is a metadata model and language recommended by the W3C for building an infrastructure of machinereadable semantics for the data on the Web, a long-term vision known as $\mathrm{Se}$ mantic Web. In the RDF model, the universe to be modeled is a set of resources, essentially anything that can have a universal resource identifier, URI. The language to describe them is a set of properties, technically binary predicates. Descriptions are statements very much in the subject-predicate-object structure. Both subject and object can be anonymous objects, known as blank nodes. In addition, the RDF specification includes a built-in vocabulary with a normative semantics (RDFS) 4. This vocabulary deals with inheritance of classes and properties, as well as typing, among other features allowing the descriptions of concepts and relationships that can exist for a community of people and software agents, enabling knowledge sharing and reuse.

Although some studies exist about addressing changes in an ontology [15], or the need for temporal annotations on Web documents [22, little attention has deserved the problem of representing, updating and querying temporal information in RDF. Time is present in almost any Web application. Indeed, as pointed out by Abiteboul 1 the modeling of time is one of the key primitives needed in a query language for Web and semistructured data. Thus, there is a clear need of applying temporal database concepts to RDF to allow metadata navigation across time. 

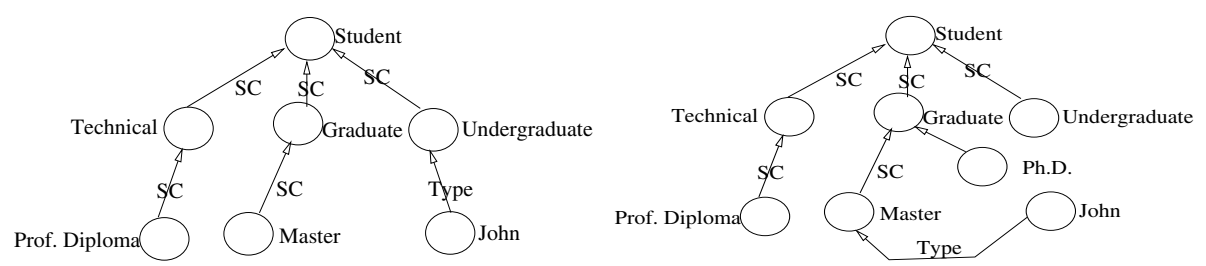

Fig. 1. Initial RDF graph (left) and after some changes (right)

Consider an RDF graph describing information about a university, as of its creation time, Figure 1 (left). Students were classified as technical, graduate or undergraduate, and the only graduate programs offered were at the level of 'Master' studies, like MBA or MSc; 'Professional Diploma' was the only program offered at the technical level. As the university evolved, a Ph.D program was created. Figure1(right) illustrates the new situation. Notice the dynamics of this example: students (e.g., John) can enroll in one program (e.g., Undergraduate), then shift to another one (e.g., Master), and so on. The figures show that the impact of disregarding the time dimension is twofold: on the one hand, when a change occurs, a new metadata document must be created (and the current document dropped). On the other hand, queries asking for past states of the metadata cannot be supported. For instance, we cannot ask for the programs offered at the time when the university was created.

\subsection{Problem Statement: Introducing Time into RDF}

Generally speaking, a temporal database is a repository of temporal information. Although temporal databases were initially studied for adding the time dimension to relational databases, as new data models emerged, temporal extensions to these models were also proposed (see Section 1.2). We next discuss the main issues that arise when extending RDF with temporal information.

Versioning Versus Time Labeling. There are two mechanisms for adding the time dimension to non-temporal RDF graphs: labeling and versioning (following the timestamp and snapshot models, respectively). The former consists in labeling the elements subject to changes (i.e. triples). The latter is based on maintaining a snapshot of each state of the graph. For instance, each time a triple changes, a new version of the RDF graph is created, and the past state is stored somewhere. Although both models are equivalent, versioning appears to be not suitable for queries of the form: "all time instants where $\Phi$ holds in the database".

There are at least two temporal dimensions to consider when dealing with temporal databases: valid and transaction times. Valid time is the time when data is valid is the modeled world; transaction time is the time when data is actually stored in the database. The versioning approach captures transaction time, while labeling is mostly used when representing valid time. The approach we present in this paper supports both time dimensions. 


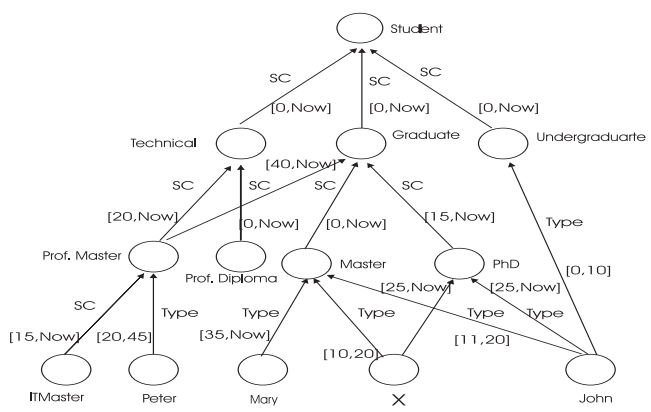

Fig. 2. A temporal RDF graph accounting for the evolution of the university ontology

In summary, we believe that for RDF data, labeling is better than versioning, because (a) it preserves the spirit of the distributed and extensible nature of $\mathrm{RDF}$, and (b) in scenarios where changes are frequent and only affecting a few elements of the document, creating a new physical version of the graph each time an update occurs may lead to large overheads when processing temporal queries that span multiple versions.

Time Points Versus Time Intervals. We will work with the point-based temporal domain for defining our data model and query language, but we will encode timepoints in intervals when possible, for the sake of clarity. We will consider time as a discrete, linearly ordered domain, as usual in virtually all temporal database applications. An ordered pair $[a, b]$ of time points, with $a \leq b$, denotes the closed interval from $a$ to $b$. Figure 2 shows a temporal RDF graph for the university example above. The arcs in the graph are labeled with their interval of validity 1 For example, the interval [0,Now] says that the triple (technical,sc,student) is valid from the document's creation time to the current time. Also, note that the figure shows that John was an Undergraduate student in the interval $[0,10]$, and now he is a PhD student.

Vocabulary for Temporal Labeling. Temporal labeling can be implemented within the RDF specification, making use of a simple additional vocabulary, as Figure 3 shows. As we adopted the point-based, discrete and linearly ordered temporal domain, the left and right hand sides of Figure 3 are equivalent. We will use both representations indistinctly. Moreover, we define constructs that allow moving between intervals and time instants as follows: the instants depicted in Figure 3 (left) can be encoded in an interval as shown in Figure 3 (right). Both alternatives will be used in the query language.

\footnotetext{
${ }^{1}$ Note that the standard graph(ical) representation of an RDF graph is not the most faithful to convey the idea of statements (triples) being labeled by a temporal element. Technically, temporal labels should be attached to a whole subgraph $u \stackrel{p}{\rightarrow} v$, and not only to an arc.
} 

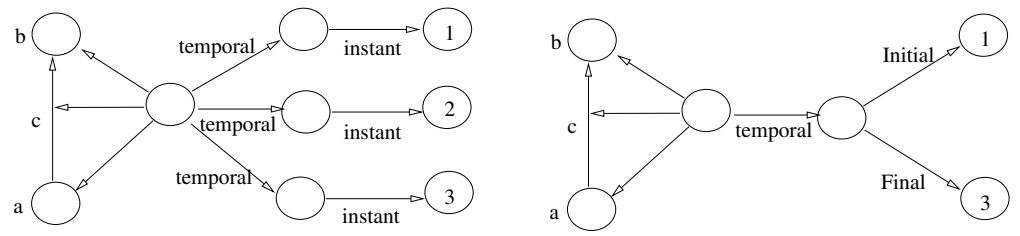

Fig. 3. Point-based labeling (left) and Interval-based labeling (right)

Temporal Entailment. An RDF graph can be regarded as a knowledge base from which new knowledge, i.e., other graphs, may be entailed. Entailment in a temporal setting is a slightly more involved in the RDF case than in the standard database case. In principle, one may be tempted to define the semantics as in temporal relational databases, i.e., defining the temporal database as the union of all of its snapshots. (A snapshot at time $t$ of a temporal RDF graph $G$ is the corresponding subgraph formed by triples labeled by and instant $t$.) Blank nodes impose some constraints to this naive approach. For example, each of the three snapshots of Figure 4 (right) entails the corresponding snapshots of Figure 4 (left). However, the whole graph of Figure 4 (left) cannot be entailed by the graph of Figure 4 (right). Indeed, the graph of Figure 4 (left) states that there is an anonymous object $X$ in the triple $(a, b, X)$ at times 3 and 4 , which is not the case for the other graph.

Temporal Query Language. Regarding query languages in temporal databases, basically two choices for defining the temporal domains exist: the point-based and the interval based temporal domains, yielding different query languages 20, 3. In the point-based approach, temporal variables in query languages refer to individual time instants, while in the interval-based domain, variables in the queries range over intervals, making queries more complicated and unnatural. Anyway, one can move easily between these two domains.

\subsection{Related Work}

The RDF model was introduced five years ago as a W3C recommendation [14. Formal work in RDF includes the study of formal aspects of RDF data and query languages [10,21, considering RDF features like the entailment, presence
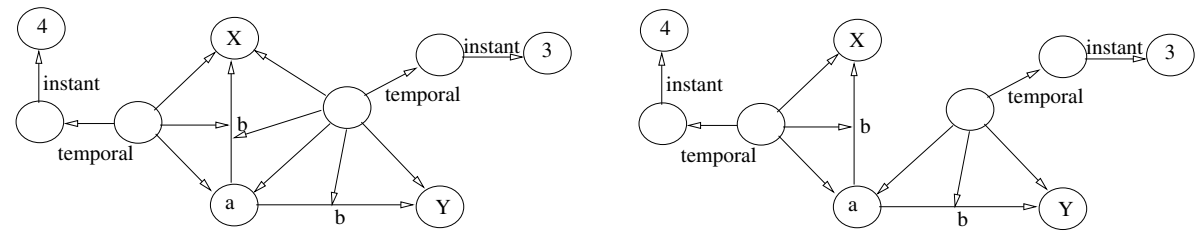

Fig. 4. Temporal entailment: for each $t$ the corresponding snapshots at $t$ are equivalent, but the graph on the left is not entailed by the graph on the right 
of blank nodes, reification, premises in queries, and the RDFS vocabulary with predefined semantics. Several languages for querying RDF data have been proposed and implemented. Some of them in the lines of traditional database query languages (e.g. SQL, OQL), others based on logic and rule languages. Good surveys are [13, 16. To the best of our knowledge, there is still no formal study of temporality issues in RDF graphs and RDF query languages.

Temporal database management has been extensively studied, including data models, mostly based on the relational model and query languages [19, leading to the TSQL2 language [18. Beyond the relational model, managing historical semistructured data was first proposed by Chawathe et al [6], who extended the Object Exchange Model (OEM) with the ability to represent updates and to keep track of them by means of "deltas." Later, Dyreson et al 7 allowed annotations on the edges of the database graph. In the XML world, Amagasa et al 2 . introduced a temporal data model based on XPath for the first time. Dyreson 8 proposed an extension of XPath with support for transaction time by means of the addition of several temporal axes for specifying temporal directions, focusing on document versioning over the web in the absence of explicit time stamps. Chien et al [5] proposed update and versioning schemes for XML through an edit-based schema in which the most current version of the document is maintained, and reverse edit scripts allow moving backward in time. Gao et al 9 introduced $\tau$ XQuery, an extension to XQuery supporting valid time while maintaining the data model unchanged. Mendelzon et al [17] proposed a temporal model for XML, a temporal extension to XPath, and a novel indexing strategy for temporal XML documents. Like in our approach, they use labeling, and a point-based temporal domain and query language. Finally, Visser et al 22 proposed a temporal reasoning framework for the Semantic Web, which has been applied in BUSTER, an ontology-based prototype developed at the University of Bremen, supporting the so-called concept@location in time type of query.

\subsection{Contributions}

In this paper we present a framework to incorporate temporal reasoning into RDF, yielding temporal RDF graphs. In particular, we present the following contributions: (a) a semantics for temporal RDF graphs in terms of the semantics of non-temporal RDF and RDFS graphs; (b)a study of properties of temporal RDF graphs and the interplay between timestamp and snapshot semantics in temporal RDF graphs; (c) a syntax to incorporate this framework into standard RDF graphs, which includes a vocabulary and rules. The syntax uses the standard RDF vocabulary plus temporal labels; (d) a sound and complete inference system for temporal RDF graphs; (e) complexity bounds which show that entailment in temporal RDF graphs does not yield extra asymptotic time complexity with respect to standard RDF graphs; (f) a sketch for a temporal query language for RDF. For the sake of space, we do not include proofs in this version of the paper. 


\section{RDF Preliminaries}

In this section we present a streamlined formalization of the RDF model following the W3C documents [14, 12, 4], along the lines of [10].

\subsection{RDF Graphs}

Assume there is an infinite set $U$ (RDF URI references); an infinite set $B=$ $\left\{N_{j}: j \in \mathbb{N}\right\}$ (Blank nodes); and an infinite set $L$ (RDF literals). A triple $\left(v_{1}, v_{2}, v_{3}\right) \in(U \cup B) \times U \times(U \cup B \cup L)$ is called an $R D F$ triple. In such a triple, $v_{1}$ is called the subject, $v_{2}$ the predicate and $v_{3}$ the object. We often denote by UBL the union of the sets $U, B$ and $L$.

An $R D F$ graph (just graph from now on) is a set of RDF triples. A subgraph is a subset of a graph. The universe of a graph $G$, universe $(G)$, is the set of elements of UBL that occur in the triples of $G$. The vocabulary of $G$ is the set universe $(G) \cap$ $(U \cup L)$. We will use letters $N, X, Y, \ldots$ to denote blank nodes, and $a, b, c, \ldots$ for URIs and literals. A graph is ground if it has no blank nodes. Graphically we represent RDF graphs as follows: each triple $(a, b, c)$ is represented by the labeled graph $a \stackrel{b}{\rightarrow} c$. Note that the set of arc labels can have non-empty intersection with the set of node labels.

A map is a function $\mu: \mathrm{UBL} \rightarrow \mathrm{UBL}$ preserving URIs and literals, i.e., $\mu(u)=u$ and $\mu(l)=l$ for all $u \in U$ and $l \in L$. Given a graph $G$, we define $\mu(G)$ as the set of all $(\mu(s), \mu(p), \mu(o))$ such that $(s, p, o) \in G$. A map $\mu$ is consistent with $G$ if $\mu(G)$ is an RDF graph, i.e. , if $s$ is the subject of a triple, then $\mu(s) \in U B$, and if $p$ is the predicate of a triple, then $\mu(p) \in U$. In this case, we say that the graph $\mu(G)$ is an instance of the graph $G$. An instance of $G$ is proper if $\mu(G)$ has fewer blank nodes than $G$. This means that either $\mu$ sends a blank node to an URI or a literal, or identifies two blank nodes of $G$. We will overload the meaning of map and speak of a map $\mu: G_{1} \rightarrow G_{2}$ if there is a map $\mu$ such that $\mu\left(G_{1}\right)$ is a subgraph of $G_{2}$.

Two graphs $G_{1}, G_{2}$ are isomorphic, denoted $G_{1} \cong G_{2}$, if there are maps $\mu_{1}, \mu_{2}$ such that $\mu_{1}\left(G_{1}\right)=G_{2}$ and $\mu_{2}\left(G_{2}\right)=G_{1}$.

We define two operations on graphs. The union of $G_{1}, G_{2}$, denoted $G_{1} \cup G_{2}$, is the set theoretical union of their sets of triples. The merge of $G_{1}, G_{2}$, denoted $G_{1}+G_{2}$, is the union $G_{1} \cup G_{2}^{\prime}$, where $G_{2}^{\prime}$ is an isomorphic copy of $G_{2}$ whose set of blank nodes is disjoint with that of $G_{1}$. Note that $G_{1}+G_{2}$ is unique up to isomorphism.

\subsection{RDFS Vocabulary}

There is a set of reserved words defined in the RDF vocabulary description language, RDF Schema [4, -just rdfs-vocabulary for us- that may be used to describe properties like attributes of resources (traditional attribute-value pairs), and also to represent relationships between resources. It defines classes and properties that may be used for describing groups of related resources and relation- 
ships between resources 2 Classes are sets of resources. Elements of a class are known as instances of that class. To state that a resource is an instance of a class, the property rdf:type may be used. The following are the most important classes (in brackets the name we will use in this paper) rdfs:Resource [res], rdfs:Class [class], rdfs:Literal [literal], rdfs:Datatype [datatype], rdf:XMLLiteral [xmlLit], rdf:Property [property]. Properties are binary relations between subject resources and object resources. The built-in properties are: rdfs:range [range], rdfs:domain [dom], rdf:type [type], rdfs:subClassOf [sc], rdfs:subPropertyOf [sp].

\section{Temporal RDF Graphs}

In this paper we extend RDF graphs by allowing temporal elements to label triples. A temporal label is a temporal element $t$ labeling a triple $(a, b, c)$. For simplicity, without loss of generality, we will work with single intervals instead of temporal elements. In an RDF graph, given a triple $(a, b, c)$, the temporal element $t$ represents the time period when the triple was valid, i.e. the valid time of the triple. At this time we do not deal with transaction time, which can be addressed in an analogous way.

\subsection{Basic Definitions}

In this section we define the notion of temporal RDF at a conceptual level.

\section{Definition 1 (Temporal graph).}

1. A temporal triple is an RDF triple with a temporal label (a natural number). We will use the notation $(a, b, c):[t]$. The expression $(a, b, c):\left[t_{1}, t_{2}\right]$ is a notation for $\left\{(a, b, c):[t] \mid t_{1} \leq t \leq t_{2}\right\}$.

2. A temporal graph is a set of temporal triples. A subgraph is a subset of the graph.

For a temporal graph $G$, define the snapshot at time $t$ as the RDF graph

$$
G(t)=\{(a, b, c) \mid(a, b, c):[t] \in G\}
$$

The underlying RDF graph of a temporal RDF graph $G$, denoted $u(G)$, is $\bigcup_{t} G(t)$, the union of the graphs $G(t)$.

For an RDF graph, define $G^{t}$ as the temporalization of all its triples by a temporal mark $t$, that is, $G^{t}=\{(a, b, c):[t] \mid(a, b, c) \in G\}$.

The above definitions give the following elementary consequences about the relationship between RDF graphs and temporal RDF graphs.

\footnotetext{
${ }^{2}$ We omit in this paper vocabulary intended to describe lists, collections, some variations on these, as well as vocabulary to help document and describe other functionalities for which there is no normative semantics. The complete vocabulary can be consulted in 4].
} 
Lemma 1. Let $G$ be an $R D F$ graph, and $G^{\prime}$ be a temporal RDF graph. Then: (1) $G^{t}(t)=G$; (2) $\left(G^{\prime}(t)\right)^{t} \subseteq G^{\prime}$, and (3) $G^{\prime}=\bigcup_{t}\left(G^{\prime}(t)\right)^{t}$.

Several issues on the definition of temporal RDF graph are in order:

- Recall we use a temporal model where an interval $[a, b]$ is of the form $[a, a+$ $1, \ldots, b]$ for a given unit of time that we will assume to be universal in this paper. The natural way to approach this issue is to specify, together with the temporal mark, the unit of time it represents. All the results given here extend without difficulties to this setting.

- Temporal triples do not belong to the RDF syntax. In the next section we introduce an RDF-complying syntax for temporal triples, using a small temporal vocabulary.

- Source of a temporal statement: Due to the extensible nature of the RDF model, it is possible to include the source of a temporal statement (i.e. who is the author of the temporal statement), and other properties that apply. Although our model (see next section) allows this, we will not study the semantic consequences of this extra information in this paper, but rather stay in the classic setting of temporal models.

\subsection{Semantics}

In what follows, we present the semantics for the notion of entailment for temporal graphs based on the corresponding notion for RDF graphs.

Definition 2 (Temporal Entailment). Let $G_{1}, G_{2}$ be RDF temporal graphs. Define

- For ground temporal RDF graphs $G_{1}, G_{2}$ define $G_{1} \models{ }_{t} G_{2}$ as $G_{1}(t) \models G_{2}(t)$ for each $t$;

- For general graphs, $G_{1} \models_{t} G_{2}$ iff there exist ground instances $\mu_{1}\left(G_{1}\right)$ and $\mu_{2}\left(G_{2}\right)$ such that $\left(\mu_{1}\left(G_{1}\right)\right)(t)=\left(\mu_{2}\left(G_{2}\right)\right)(t)$ for each $t$.

Note that the definition for ground graphs resembles classical temporal definitions:

Proposition 1. Let $G_{1}, G_{2}$ be temporal graphs. Then, $G_{1} \models_{t} G_{2}$ implies $G_{1}(t) \models$ $G_{2}(t)$ for all $t$, and the converse is true for ground graphs.

In fact, the problems for general graphs are introduced by blank nodes and the notion of entailment. For example, $G_{1}(t) \models G_{2}(t)$ for all $t$ does not imply $G_{1} \models_{t} G_{2}$ (see Figure 4). We have the following issues:

- A blank node represents the same (unnamed) resource throughout the time range, rather than a sequence of different resources. This makes the behavior of temporal marks in Temporal RDF different from the classical setting. Temporal marks here -contrary to temporal XML for example- are not only a relation among fixed objects, but also among time-varying objects, the blank nodes. See example in Figure 4 
- The notion of entailment for temporal RDF needs a basic arithmetic of intervals in order to combine the notion of temporality and deductive properties. For example if we have $(a, \mathrm{sc}, c):[2,3],(c, \mathrm{sc}, d):[2]$, then we should be able to derive $(a, \mathrm{sc}, d):[2]$, but not $(a, \mathrm{sc}, d):[3]$.

In the rest of this section, we show that the notions of closure, lean graph, core -fundamental to define notions of normalization of this data- can be extended without difficulty to the temporal setting. (Compare discussion in [10]).

The closure of a temporal graph $G$, denoted $\operatorname{tcl}(G)$, is a maximal set of temporal triples $G^{\prime}$ over universe $(G)$ plus the RDF vocabulary such that $G^{\prime}$ contains $G$ and is equivalent to it.

\section{Proposition 2 (Entailment for Temporal graphs).}

Let $G, G_{1}, G_{2}$ be temporal $R D F$ graphs. Then

1. $\operatorname{tcl}(G)=\bigcup_{t}(\operatorname{cl}(G(t)))^{t}$;

2. $G_{1} \models{ }_{t} G_{2}$ if and only if $\operatorname{tcl}\left(G_{1}\right) \models_{t} G_{2}$.

A temporal graph $G$ is lean if and only if there is no proper temporal subgraph $G^{\prime}$ of $G$ such that $G \models_{t} G^{\prime}$. The core of $G$, core $(G)$ is a lean subgraph of $G$ equivalent to it. With these notions, for a temporal RDF graph $G$ we can define -as in the case of RDF graphs- a notion of normal form, denoted by $\operatorname{nf}_{t}(G)$, as follows: $\operatorname{nf}_{t}(G)=\operatorname{core}_{t}\left(G^{\prime}\right)$ for a temporal closure $G^{\prime}$ of $G$.

The computational complexities of computing the core and testing whether a graph is lean, are asymptotically the same as the case of standard RDF graphs [10].

Proposition 3. Let $G, G^{\prime}$ be graphs.

1. The problem of deciding if $G^{\prime}$ is the closure of $G$ is DP-complete.

2. The problem of deciding if $G^{\prime}$ is the normal form of $G$ is DP-complete.

\section{Syntax and Deductive System for Temporal Graphs}

We present a deductive system for temporal RDF. It is based on a sound and complete set of rules given in [12, plus three rules capturing temporal issues.

\subsection{RDF Syntax of Temporal Triples}

Definition 3 (Temporal vocabulary). The temporal vocabulary is the following: temporal (abbreviated as tpl), instant,interval, initial and final, all of type property, and now of type plain literal. The range of instant, initial and final is the set of natural numbers.

We will use the following notation shortcuts: $\operatorname{reif}(a, b, c, X)$ : the set of triples $(X$, tsubj, $a),(X$, tpred,$b),(X$, tobj,$c)$ (a kind of "temporal reification" of $(a, b, c)) .^{3}$

\footnotetext{
${ }^{3}$ We could have used here the standard reification vocabulary of RDF. We chose not to in order to stress the fact that the notions presented in this paper are independent of any view one may have about the concept of reification in RDF.
} 
Definition 4 (Temporal triples and graphs). Temporal triples are the following graphs using the temporal vocabulary.

- $(a, b, c), \operatorname{reif}(a, b, c, X),(X, \mathrm{tpl}, Y),(Y$, instant, $n)$ where $n$ is a natural number; we will summarize this as $(a, b, c):[X, Y, n]$;

- $(a, b, c), \operatorname{reif}(a, b, c, X),(X, \operatorname{tpl}, Y)$, (Y, interval, $Z)$, ( $Z$, initial, $I)$, $(Z$, final, $F)$; where $I, F$ are natural number; we will summarize this as $(a, b, c):[X, Y, I, F]$

A temporal graph will be defined as a merge of a set of temporal triples.

Because RDF is extensible, nothing prevents the use of the blank nodes included in the definition as target or source of other properties beyond the temporal vocabulary. We want to have a definition of temporal triple independent of the blank nodes occurring in the proposed syntactic definition of temporal triples, e.g., we want that $(a, b, c):[X, Y, n]$ be essentially equivalent to $(a, b, c):[n]$. Both previous issues are overcame in our syntax by adding certain rules, which regulate the temporal vocabulary.

\subsection{Rules}

The set of rules is arranged in four groups. Groups A, B, C, and D are intended to describe the classical RDFS semantics, and we follow the approach in 10 . We omit another group of rules that has to do with internal relationships of the RDF model itself and that we do not consider in this paper.

The novelty here is Group $\mathrm{T}$ (temporal rules), whose main objective is to be able to standardize the interval version and the instant version as well as help defining "absolute" temporal marks.

GROUP A (Existential) For a map $\mu: G^{\prime} \rightarrow G, \quad \frac{G}{G^{\prime}}$

GROUP B (Subproperty)

$$
\frac{(a, \text { type }, \text { property })}{(a, \mathrm{sp}, a)}, \quad \frac{(a, \mathrm{sp}, b)(b, \mathrm{sp}, c)}{(a, \mathrm{sp}, c)}, \quad \frac{(a, \mathrm{sp}, b)(x, a, y)}{(x, b, y)}
$$

GROUP C (Subclass)

$$
\frac{(a, \text { type }, \mathrm{class})}{(a, \mathrm{sc}, a)}, \quad \frac{(a, \mathrm{sc}, b)(b, \mathrm{sc}, c)}{(a, \mathrm{sc}, c)}, \quad \frac{(a, \mathrm{sc}, b)(x, \text { type }, a)}{(x, \text { type }, b)}
$$

GROUP D (Typing)

$$
\frac{(a, \text { dom }, c)(x, a, y)}{(x, \text { type }, c)} \quad \frac{(a, \text { range }, d)(x, a, y)}{(y, \text { type }, d)}
$$

\section{GROUP T (Temporal)}

$$
\begin{gathered}
(i 2 t) \frac{\left\{(X, \mathrm{tpl}, Y),(Y, \text { instant } n): n \in\left[t_{1}, t_{2}\right]\right\}}{(X, \mathrm{tpl}, Y),(Y, \text { int }, Z),\left(Z, \text { initial }, t_{1}\right),\left(Z, \text { final }, t_{2}\right)} \\
(t 2 i) \frac{(X, \mathrm{tpl}, Y),(Y, \text { int }, Z),\left(Z, \text { initial }, t_{1}\right),\left(Z, \mathrm{final}, t_{2}\right)}{\{(X, \mathrm{tpl}, Y),(Y, \text { instant }, n)}, n \in\left[t_{1}, t_{2}\right]
\end{gathered}
$$




$$
(a b s) \frac{(a, b, c):\left[X_{1}, Y_{1}, n_{1}\right],(a, b, c):\left[X_{2}, Y_{2}, n_{2}\right]}{(a, b, c):\left[U, V, n_{1}\right],(a, b, c):\left[U, V, n_{2}\right]}, U, V \text { fresh }
$$

Rules (i2t) (interval to instants) and (t2i) are needed to standardize the interval version and the instant version, by making them equivalent. Rule (abs) essentially says that marks (instants) can be collected in a single node. This permits to concentrate on temporal marks independent of other contexts in which the variables involving temporal vocabulary are immersed.

The definition behaves well in the sense of the following lemma.

Lemma 2. 1. There exist blanks $X, Y$ such that $G \models_{t}(a, b, c):\left[X, Y, t_{1}, t_{2}\right]$ if and only if there exist blanks $U, V$ such that $\forall j$ with $t_{1} \leq j \leq t_{2} G \models_{t}$ $(a, b, c):\left[U, V, t_{j}\right]$

2. There exist blanks $X_{1}, Y_{1}, X_{2}, Y_{2}$ such that $G \models_{t}(a, b, c):\left[X_{1}, Y_{1}, t_{1}\right]$ and $G \models_{t}(a, b, c):\left[X_{2}, Y_{2}, t_{2}\right]$ if and only if there exist blanks $U, V$ such that $G \models_{t}(a, b, c):\left[U, V, t_{1}\right]$ and $G \models_{t}(a, b, c):\left[U, V, t_{2}\right]$.

For a temporal RDF graph $G$, define $G^{*}$ as the $\operatorname{RDF}$ graph $\{(a, b, c)$ : $\left.\left[X_{t}, Y_{t}, t\right] \mid(a, b, c):[t] \in G\right\}$, where $X_{t}, Y_{t}$ are free blank variables, different for each $t$. Conversely, for each RDF graph $G$ with temporal vocabulary, define $G_{*}$ as the temporal graph defined as $\{(a, b, c):[t] \mid \exists X \exists Y(a, b, c):[X, Y, t] \in G\}$.

Theorem 1. 1. Let $G_{1}, G_{2}$ be temporal RDF graphs. Then $G_{1} \models_{t} G_{2}$ implies $G_{1}^{*}=G_{2}^{*}$.

2. Let $G_{1}, G_{2}$ be RDF graphs with temporal vocabulary. Then $G_{1} \models G_{2}$ implies $\left(G_{1}\right)_{*} \models_{t}\left(G_{2}\right)_{*}$.

3. Let $G$ be a temporal $R D F$ graph, and $G^{\prime}$ an RDF graph with temporal vocabulary. Then $\left(G^{*}\right)_{*}=G$ and $G^{\prime}=\left(G_{*}^{\prime}\right)^{*}$.

Now we can show that the syntax introduced captures the semantics of temporal RDF. The following deductive system based on the rules presented, is sound and complete for entailment of RDF graphs with rdfs vocabulary.

Definition 5. Let $G$ be a graph. For each rule $r: \frac{A}{B}$ above, define $G \vdash_{r} G \cup \mu(B)$ iff there is a map $\mu: A \rightarrow G$. Also define $G \vdash_{s} G^{\prime}$ if and only if $G^{\prime}$ is a subgraph of $G$.

Define $G \vdash G^{\prime}$ if there is a finite sequence of graphs $G_{1}, \ldots, G_{n}$ such that (1) $G=G_{1}$; (2) $G^{\prime}=G_{n}$; and (3) for each i, either, $G_{i} \vdash_{r} G_{i+1}$ for some $r$, or $G_{i} \vdash_{s} G_{i+1}$.

The following theorem shows that one can give a syntactic characterization over RDF graphs with temporal vocabulary for entailment of temporal RDF graphs:

Theorem 2. For any pair of temporal $R D F$ graphs $G_{1}, G_{2}$ :

$G_{1} \models{ }_{t} G_{2}$ if and only if $G_{1}^{*} \vdash G_{2}^{*}$. 
Note that that we cannot establish the theorem in its complete generality, namely, to prove that for RDF graphs $G_{1}, G_{2}$ with temporal vocabulary, $G_{1} \vdash G_{2}$ if and only if $\left(G_{1}\right)_{*} \models_{t}\left(G_{2}\right)_{*}$. (Both graphs in Figure 4 have identical ( ) * $_{\text {-images }}$ but are not $\vdash$-equivalent.)

The previous theorem permits to concentrate for the following sections in temporal RDF (instead of diving into syntactic issues).

\section{Query Language}

In this section we present query language for temporal RDF graphs, along with its semantics. We also present a brief study of the complexity of query processing.

\subsection{The Query Language by Example}

We will give the flavor of the query language using our running example, the database of Figure 2, Let us begin with a simple query: "Find students who have taken a Master course between year 2000 and now and return them qualified by 21-century-student". This query can be expressed as:

(?X, type, 21-century-student) $\longleftarrow$

$(? X$, takes, $? C):[? T],(? C$, type, Master $):[? T], 2000 \leq ? T, ? T \leq$ Now.

This example query illustrates the need of a built-in arithmetic language in order to reason about time and intervals. Another important observation is that temporal queries may output non-temporal RDF graph, as the previous query does.

For the query asking for a snapshot of the graph at $t_{1}$, we have:

$$
(? X, ? Y, ? Z) \leftarrow(? X, ? Y, ? Y):\left[t_{1}\right]
$$

Now consider the query "Students taking Ph.D courses together, and the time instants when this occurred." For simplicity we expressed this as a point-based query. The translation of the result into intervals is straightforward.

$$
(? \mathrm{X}, \text { together, } ? \mathrm{Y})[? T] \leftarrow(? \mathrm{X}, \text { type, Ph.D) }:[? T],(? \mathrm{Y}, \text { type, Ph.D) : [?T]. }
$$

Next, we give examples of queries that use temporal triples with intervals. The query "Time intervals when the IT Master was offered" can be expressed as follows:

$$
\begin{aligned}
(X, \text { interval, } Y),\left(Y, \text { initial, } T_{i}\right),\left(Y, \text { final, } T_{f}\right) & \leftarrow \\
& (\text { IT Master, sc, Prof.Master }):\left[T_{i}, T_{f}\right] .
\end{aligned}
$$

Observe that the previous query returns a set of intervals. In order to retrieve maximal intervals we need a more subtle query, since their computation do not 
follow from the temporal rules. For the query "Compute the maximal interval when the triple $(a, b, c)$ holds", we need aggregate operators MAX and MIN.

$$
(a, b, c):\left[? T_{1}, ? T_{2}\right] \leftarrow(a, b, c):\left[? T_{i}, ? T_{f}\right], ? T_{1}=\operatorname{MIN}\left(? T_{i}\right), ? T_{2}=\operatorname{MAX}\left(? T_{f}\right)
$$

For a query asking for "Students applying for jobs at time $t$ after finishing their Ph.D. program in no more than 4 years", we have:

$$
(? X \text {, apply, job }) \leftarrow(X, \text { type, Ph.D }):\left\|t_{i}, t_{f}\right\|, t_{f}-t_{i}<4, t_{f}<t .
$$

Here, the notation $\left\|t_{i}, t_{f}\right\|$ stands that $t_{i}$ and $t_{f}$ match with the maximal interval for the corresponding triple computed with the query given above.

\subsection{Semantics and Complexity}

Let $V$ be a set of variables (disjoint from UBLT). Individual variables will be denoted ?X, ?Y, ?Z, etc. There is also a set of temporal variables $V_{t} \subset V$.

The query language we define is analogous to the one presented by Gutierrez et al. 11]. A query is a temporal tableau, which is a pair $(H, B \cup A)$, where $H$ and $B$ are temporal RDF graphs with some elements of UBL replaced by variables in $V$, and with some elements of $\mathrm{T}$ replaced with variables in $V_{t}, B$ has no blank nodes and all the variables in $H$ occur also in $B$. The set $A$ has the usual arithmetic built-in predicates such as $\langle\rangle,,=$, . over elements in $V_{t}$ and $T$.

We adopt the usual notion of safe rule from Datalog to prevent operations on infinite predicates. A rule is safe if all its variables are limited. A variable is limited if one of the following hold: a variable appears as an argument in a non-built-in predicate of the body; the variable $X$ appears in a subgoal $X=t$ (or $t=X$ ), where $t$ is a constant in $T$; or the variable $X$ appears in a subgoal $X=Y($ or $Y=X)$, where $Y$ is limited.

The semantics is the usual in these cases. Given a temporal tableau $(H, B \cup A)$ and a temporal RDF graph $G$, for each matching of the graph pattern $B$ in $G$, pick up the values of the variables and check whether they satisfy the built-in predicates in $A$. If this is the case, construct a pre-answer, which is the graph resulting by substituting the values of the variables in the head. Finally, the answer of the query is the union of all pre-answers.

We end this section by showing that the additional time dimension in our model does not play any relevant role in the complexity of query answering, that is, the query language preserves the tractability of answers. In order to do this, we consider the simpler problem of testing emptiness of the query answer set in the following forms: (1) Query complexity version: For a fixed database $D$, given a query $q$, is $q(D)$ non-empty? (2) Data complexity version: For a fixed query $q$, given a database $D$, is $q(D)$ non-empty?

Theorem 3. The evaluation problem is NP-complete for the query complexity version, and polynomial for the data complexity version.

The previous result shows that the temporal labeling over the triples does not introduce any complexity overhead. This is consistent with previous works 
in temporal databases. As Toman [20] showed, a point-based temporal query language has the same properties than a first order query language, in spite of the temporal variable.

\section{Conclusions}

We have proposed an RDF vocabulary to assert the times when triples are valid in RDF graphs. This allows an explicit treatment of time inside RDF. We have also offered a complete and sound inference procedure for temporal RDF graphs, and a query language for them. Our framework allows to browse, query, and reason across different versions of RDF graphs.

There are several aspects left for future work. Among the most important are the definition of a built-in arithmetic, aggregate functions, and a unified semantic for the two classes of RDF answers - temporal and plain- which would allow closeness and full query composition in a temporal query language for RDF. Probably one of the challenging issues open is the handling of anonymous times. For example, one may want to say that a triple holds in a particular time inside an interval, but do not know the exact valid time of the triple. Anonymous times may help in the specification of triples without temporal labels, which is a form to specify incomplete temporal information.

Acknowledgments. This research was supported by Millennium Nucleus, Center for Web Research (P01-029-F), Mideplan, Chile. C. Gutierrez and C. Hurtado were supported by FONDECYT 1030810.

\section{References}

1. S. Abiteboul. Querying Semi-Structured Data. Proceedings of the 6th International Conference on Database Theory (ICDT'97). Delphi, Greece, 1997.

2. T. Amagasa, M. Yoshikawa, S. Uemura, A Temporal Data Model for XML Documents, Proceedings of DEXA Conference, 2000, 334-344.

3. M. Bölen98, R. Busatto, C.S. JensenPoint- Versus Interval-based Temporal Data Models, Proceedings of IEEE/ICDE, 1998.

4. Dan Brickley, R.V. Guha Eds., RDF Vocabulary Description Language 1.0: RDF Schema, W3C Working Draft 23 January 2003.

5. S. Chien, V. Tsotras, C. Zaniolo, Efficient Management of Multiversion Documents by Object Referencing, Proceedings of the 27th International Conference on Very Large Data Bases,2002,Rome, Italy, 291-300.

6. S. Chawathe, S. Abiteboul, J. Widom, Managing Historical Semistructured Data,Theory and Practice of Object Systems, Vol 5(3), 1999,143-162.

7. C.E. Dyreson, M.H. Bolen, C.S. Jensen, Capturing and Querying Multiple Aspects of Semistructured Data, Proceedings of the 25th VLDB Conference, 1999, 290-301.

8. C.E. Dyreson, Observing Transaction-time Semantics with TTXPath, Proceedings of WISE 2001, 2001,193-202.

9. C. Gao, R.Snodgrass, Temporal Slicing in the Evaluation of XML Queries, Proc. 29th Int. Conference on Very Large Data Bases, 2003, 632-643, Berlin, Germany. 
10. C. Gutierrez, C. Hurtado, A.O. Mendelzon, Formal aspects of querying RDF databases, Proc. SWDB 2003, 293-307.

11. C. Gutierrez, C. Hurtado, A.O. Mendelzon,Foundations of Semantic Web Databases, 23rd. Symp. on Principles of Database Systems, PODS 2004, 95-106.

12. Patrick Hayes Ed., RDF Semantics, W3C Working Draft, 1 October 2003

13. P. Haase HAASE, J. Broekstra, A. Eberhart, R. Volz. A comparison of RDF Query Languages. International Semantic Web Conference, 2004.

14. O. Lassila, R. Swick Eds., Resource description framework (RDF) model and syntax specification, Working draft, W3C, 1998.

15. A. Maedche, B. Motik, L. Stojanovic, R. Studer, R. VolzEstablishing the semantic web 11: An infrastructure for searching, reusing, and evolving distributed ontologies, Proc. of the 12th. Int. Conference on World Wide Web,2003, 439-448.

16. A. Magkanaraki et al. Ontology Storage and Querying, Technical Report No. 308, April 2002, Foundation for Research and Technology Hellas, Institute of Computer Science, Information System Laboratory.

17. A.O. Mendelzon, F. Rizzolo, A. Vaisman, Indexing Temporal XML, Proc. 30th Int. Conference on Very Large Data Bases, Toronto, Canada, 2004, 216-227.

18. R. Snodgrass, The TSQL2 Temporal Query Language, Kluwer Academic Publishers, 1995.

19. A. Tansel, J. Clifford, S. Gadia Eds.,Temporal Databases: Theory, Design and Implementation, Benjamin/Cummings, 1993.

20. D. Toman, Point vs. Interval-based Query Languages for Temporal Databases, 15 th. Symposium on Principles of Database Systems, PODS 1996, 58-67.

21. G. Yang, M. Kifer, On the Semantics of Anonymous Identity and Reification Proc. First International Conference on Ontologies, Databases and Applications of Semantics (ODBASE), 2002, 1047-1066.

22. Ubbo Visser, Intelligent Information Integration for the Semantic Web, Lecture Notes in Artificial Intelligence Volume 3159, Springer-Verlag, 2004. 